\title{
Bigger Than His Bite
}

\author{
Christopher Petrilli, MD¹, Patrick Pilie, MD¹, Sanjay Saint, MD, MPH ${ }^{1,2}$, Daniel Kaul, MD , Andrew Odden, MD ${ }^{1,2 \star}$ \\ ${ }^{1}$ Department of Internal Medicine, University of Michigan, Ann Arbor, Michigan; ${ }^{2}$ VA Ann Arbor Healthcare System, Ann Arbor, Michigan.
}

\begin{abstract}
A 58-year-old male presented to a local community hospital emergency department with fever and altered mental status. Earlier in the day he had complained of chills, swollen tongue, numbness and tingling in his extremities with associated burning pain, and generalized weakness. En route to the emergency department, he was extremely agitated and moving uncontrollably. On arrival, he was noted to be in respiratory distress and was intubated for hypoxic respiratory failure. He was subsequently transferred to an academic medical center, and in transit was noted to have sustained supraventricular tachycardia with a heart rate of 160 beats per minute.
\end{abstract}

Although the differential for altered mental status is broad, associated fever limits the main diagnostic considerations to infectious, toxic, and some inflammatory disorders. Confusion and fever are most concerning for a central nervous system infection, either meningitis or encephalitis. Sepsis from a broader range of infectious etiologies may also present with these symptoms. His respiratory failure could represent acute respiratory distress syndrome (ARDS) due to sepsis, aspiration, or a manifestation of a multisystem inflammatory disease.

He did not have any significant past medical or surgical history. Three days before his initial presentation, the patient was bitten on the left hand and forearm while breaking up a dogfight. The dogs that bit him belonged to his son, but were unvaccinated. He did not seek medical attention and it was unclear how he treated his wounds at home.

Dogs may serve as vectors for a number of zoonoses. Species of both Pasteurella and Capnocytophaga may cause sepsis and rarely meningitis as a consequence of dog bites. The incubation period of 3 days, though brief, does not exclude either infection. Rabies encephalitis is also possible, particularly given the dogs' unvaccinated status. However, the typical incubation period for rabies is on the order of months, and a 3-day interval from inoculation to symptoms would be highly unusual. Although other explanations for his symptoms are more likely, he should still be considered for vaccination and rabies immune globulin. The dogs should be observed for clinical manifestations of rabies. Despite the patient's history of dog bite, a broad differential diagnosis must be maintained.

\footnotetext{
*Address for correspondence and reprint requests: Andrew Odden, MD, VA Ann Arbor Healthcare System, Medicine Administration, B744 (111), 2215 Fuller Road, Ann Arbor, Ml 48105; Telephone: 734-845-5922; Fax: 734-913-0883; E-mail: aodden@med.umich.edu

Additional Supporting Information may be found in the online version of this article.

Received: July 31, 2014; Revised: September 5, 2014; Accepted: September 23, 2014

2014 Society of Hospital Medicine DOI 10.1002/jhm.2272

Published online in Wiley Online Library (Wileyonlinelibrary.com).
}

The patient lived in Michigan and worked in a chemical factory driving equipment without any hazardous exposures. He did not have any allergies. He drank 6 beers per day; he did not smoke cigarettes and had no history of illicit drug use. He was single and had 4 adult children.

His history of heavy alcohol consumption raises several additional possibilities. Delirium tremens, alcohol withdrawal seizures, or hepatic encephalopathy as a consequence of alcoholic cirrhosis are both potential contributors to his presentation. Furthermore, the physiologic signs of alcohol withdrawal are similar to many critical illnesses, which may present a diagnostic challenge. The patient's history of employment at a chemical factory is intriguing, though the details of any potential occupational exposures are unknown. Carbon monoxide poisoning can present with altered mental status and agitation, whereas anticholinergic toxicity can present with fever, tachycardia, and altered mental status; however, there is no obvious source of exposure to either.

On physical examination, the patient was intubated with a Glasgow Coma Scale of 11 without sedation; serial examinations revealed a fluctuating level of consciousness. His temperature was $38.1^{\circ} \mathrm{C}$, heart rate was 158 beats per minute, and blood pressure was $93 / 68 \mathrm{~mm} \mathrm{Hg}$. Mechanical ventilation was provided with assist control mode, a respiratory rate of 28 breaths per minute, tidal volume $466 \mathrm{~mL}$, and positive end expiratory pressure of $20 \mathrm{~cm}$ of water. His oxygen saturation was $81 \%$ on $100 \%$ oxygen. Examination of his neck exhibited a large left neck hematoma from the unsuccessful placement of an external jugular intravenous catheter. Pupils were $4 \mathrm{~mm}$ in diameter and minimally reactive. There was no scleral icterus. Cardiac exam revealed tachycardia and regular rhythm without murmurs, rubs, or gallops. Lung exam was significant for bilateral rhonchi and minimal tracheal secretions. Extremity exam revealed 0.25 to $1.5 \mathrm{~cm}$ in diameter puncture bite marks with abrasions on his left third and fourth upper extremity digits as well as on his left forearm. Skin exam was diffusely cool with a mottled appearance. Neurologic exam revealed absent deep tendon reflexes throughout and apparent flaccid paralysis of all 4 extremities. Examination of the abdomen, lymph nodes, mouth, and throat were unremarkable.

The shock associated with sepsis is typically distributive, with intense vasodilation that classically leads to warm extremities. His mottled, cool extremities raise concern for disseminated intravascular coagulation (DIC), which can be seen in patients with septic shock, particularly cases caused by meningococcal disease and Capnocytophaga infections. His neurologic examination is typical of lower motor neuron disease, although acute upper motor neuron lesions can also be associated with hyporeflexia. Rabies can manifest as flaccid paralysis, but this would classically predate the mental status changes. Rabies remains a consideration, albeit a 
less likely one. Zoonoses, particularly Capnocytophaga and Pasteurella, are possible; however, a thorough search for other infections leading to sepsis is still indicated. His lung findings suggest severe ARDS.

The white blood cell count was $5,900 / \mathrm{mm}^{3}$, with $91 \%$ neutrophils, $6.6 \%$ lymphocytes, and $0.5 \%$ monocytes. The hemoglobin level was $13.0 \mathrm{~g} / \mathrm{dL}$, and the platelet count was $12,000 / \mathrm{mm}^{3}$. The fibrinogen level was $89 \mathrm{mg} / \mathrm{dL}$ (normal range $200-400 \mathrm{mg} / \mathrm{dL}$ ), international normalized ratio and partial-thromboplastin time were 4.6 (normal range 0.8 to 1.1) and greater than 120.0 seconds (normal range 25-35 seconds), respectively. Lactate dehydrogenase level was 698 IU/L (normal 120-240 IU/L), and haptoglobin was $54 \mathrm{mg} /$ dL (normal 41-165 mg/dL). Serum sodium was $136 \mathrm{mmol} / \mathrm{L}$, potassium $4.6 \mathrm{mmol} / \mathrm{L}$, chloride $101 \mathrm{mmol} / \mathrm{L}$, bicarbonate 16 mmol/L, blood urea nitrogen $29 \mathrm{mg} / \mathrm{dL}$, creatinine $2.28 \mathrm{mg} /$ $\mathrm{dL}$, glucose $123 \mathrm{mg} / \mathrm{dL}$, calcium $7.0 \mathrm{mg} / \mathrm{dL}$, magnesium $1.7 \mathrm{mg} / \mathrm{dL}$, and phosphorus $7.2 \mathrm{mg} / \mathrm{dL}$. Total protein was $4.3 \mathrm{~g} / \mathrm{dL}$ (normal 6.0-8.3 g/dL), albumin $2.5 \mathrm{~g} / \mathrm{dL}$ (normal $3.5-4.9 \mathrm{~g} / \mathrm{dL}$ ), total bilirubin $2.3 \mathrm{mg} / \mathrm{dL}$ (normal $0.2-1.2 \mathrm{mg}$ / $\mathrm{dL}$ ), aspartate aminotransferase 71 IU/L (normal 8-30 IU/L), alanine aminotransferase $29 \mathrm{IU} / \mathrm{L}$ (normal 7-35 IU/L), and alkaline phosphatase 107 IU/L (normal 30-130 IU/L). The serum troponin-I level was $0.76 \mathrm{ng} / \mathrm{mL}$, creatine phosphokinase $397 \mathrm{ng} / \mathrm{mL}$, and creatine kinase-myocardial band 3.5 $\mathrm{ng} / \mathrm{mL}$. Initial arterial blood gas analysis revealed a $\mathrm{pH}$ of 7.00, pCO2 $57 \mathrm{~mm} \mathrm{Hg}$, pO2 $98 \mathrm{~mm} \mathrm{Hg}$, and a lactic acid of $6.5 \mathrm{mmol} / \mathrm{L}$ (normal $0.5-2.2 \mathrm{mmol} / \mathrm{L}$ ).

The patient has a normal absolute white blood cell count in the setting of septic shock. He has a relative neutrophilia and a marked leukopenia, both of which can be seen in overwhelming infections. The patient's arterial blood gas analysis indicates he has a mixed metabolic and respiratory acidosis. The normal physiologic response to metabolic acidosis is to increase minute ventilation and induce a compensatory respiratory alkalosis. His concomitant respiratory acidosis in the face of mechanical ventilation and presumed adequate minute ventilation suggests severely impaired alveolar gas exchange, most likely from ARDS. He has numerous other metabolic abnormalities, including acute kidney injury, DIC, and hemolytic anemia, all of which may be seen with severe bacterial infections or septic shock. Neisseria meningitidis and other gram-negative infections would be of particular concern in this case. The combination of fever, altered mental status, thrombocytopenia, hemolytic anemia, and renal failure could be consistent with thrombotic thrombocytopenic purpura. However, the prolonged coagulation studies are much more consistent with DIC.

Intravenous antimicrobials were administered including ceftriaxone (initiated in the emergency department of the transferring hospital), ampicillin, vancomycin, piperacillin/ tazobactam, clindamycin, metronidazole, doxycycline, and acyclovir. He received tetanus and rabies vaccines as well as tetanus and rabies immune globulin. The patient was given aggressive intravenous crystalloid fluids with minimal response in blood pressure. Intravenous norepinephrine was initiated to maintain a mean arterial pressure above $65 \mathrm{~mm}$ Hg. A plain chest radiograph (Figure 1) revealed perihilar airspace opacities. Head computed tomography without contrast revealed global cerebral volume loss greater than expected for the patient's age; no evidence of intracranial hemorrhage, mass effect, or edema; and proptosis of the

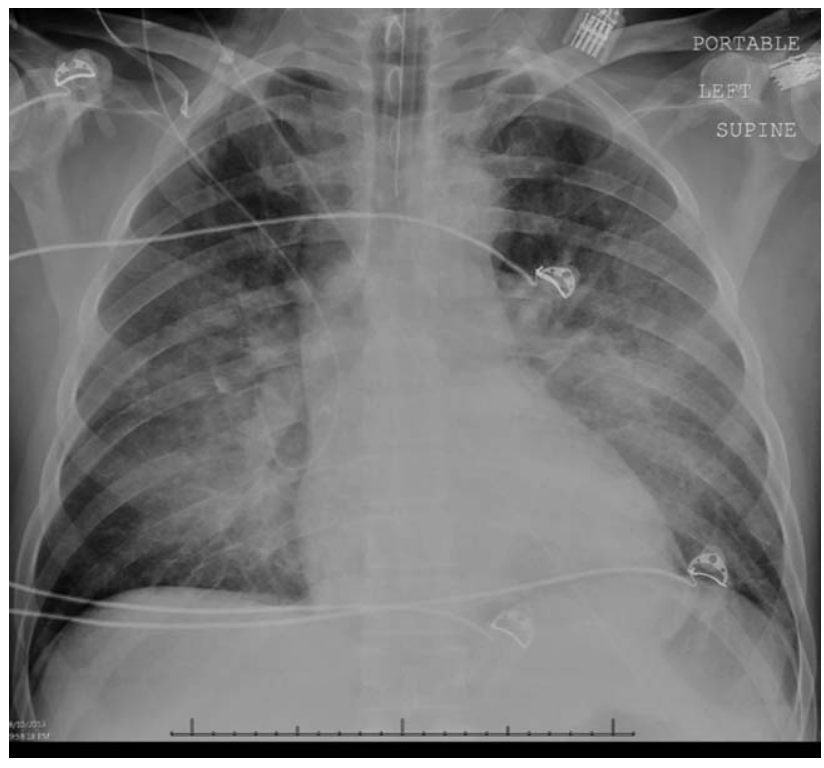

FIG. 1. Plain radiograph of the chest on presentation that revealed perihilar airspace opacities, which likely represented moderate to severe pulmonary edema.

eyes with adjacent preseptal soft tissue swelling without evidence of retrobulbar hemorrhage or vascular engorgement. Ultrasound of the left neck hematoma was negative for pulsatile mass. Electrocardiogram (ECG) revealed sinus tachycardia without evidence of ischemic changes. A bedside transthoracic echocardiogram showed hyperdynamic changes without evidence of hypokinesis but with inspiratory collapse of the inferior vena cava. Abdominal ultrasound was normal. Plain radiographs of the left hand (Figure 2) identified only mild soft tissue swelling over the dorsum of the hand. An ultrasound of the left hand and left forearm did not identify any abnormal fluid collection. A dialysis catheter was placed after the patient received platelets and fresh frozen plasma for initiation of continuous renal replacement therapy.

Given this patient's fulminant presentation, he was appropriately started on a very broad anti-infective regimen. Although fungal infections are less likely, his current

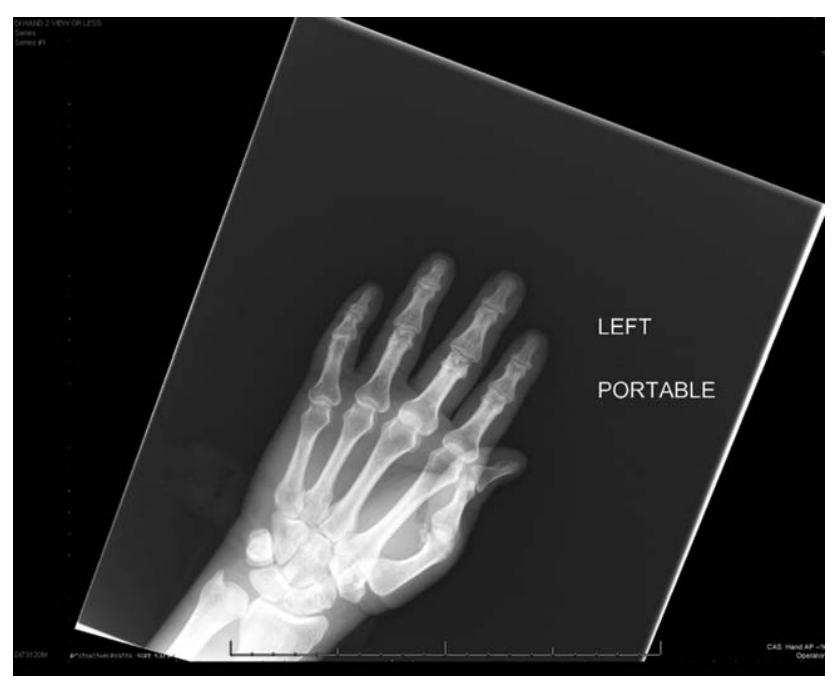

FIG. 2. Plain radiograph of the left hand that demonstrated mild soft tissue swelling over the dorsum of the hand. 
antimicrobial regimen lacks antifungal coverage. His finding of proptosis raises concern for mucormycosis, although the time course and clinical presentation are somewhat atypical. Because of the severity of his presentation, initiation of amphotericin B could be considered if he fails to quickly respond to the current regimen. There is no known effective treatment for rabies. Thus, if his presentation is due to rabies encephalitis, rabies vaccine and immunoglobulin will not be effective at treating active rabies infection. However, given his exposure history and the dogs' unvaccinated status, postexposure prophylaxis was appropriate to prevent future development of rabies. The inspiratory collapse and hyperdynamic ventricular response seen on his bedside echocardiogram is consistent with decreased effective circulating volume from sepsis or severe hypovolemia rather than acute heart failure.

Less than 36 hours after admission (60 hours after his symptoms began), the patient's oxygenation status had not improved. He developed diffuse cutaneous purpura with hemorrhagic bullae. Liver, renal, and cardiac function markers were all markedly abnormal. All cultures from the transferring hospital, collected before antibiotics were initiated, were negative to date. However, Gram stain of blood cultures performed at the academic medical center revealed "possible gram-negative rods." The patient remained unresponsive without sedation. ECG revealed evidence of inferior and anterolateral ischemia. The patient's family was informed of his persistently deteriorating condition and elected to pursue comfort measures. Two hours later the patient expired. The family agreed to an autopsy.

This patient succumbed to overwhelming sepsis and multiorgan failure. Although the etiologic pathogen is not immediately clear, several clues point to a likely unifying diagnosis. First, he has a history of a recent dog bite with minimal local evidence of infection. Second, he presented with fulminant sepsis with DIC, hemolytic anemia, and diffuse mottling that progressed to purpura fulminans. Third, a possible gram-negative rod was isolated on blood Gram stain. Fourth, he has a history of heavy alcohol use. For these reasons, Capnocytophaga canimorsus is the most likely underlying etiology. C canimorsus is a fastidious gram-negative coccobacillus that is an uncommon cause of fulminant sepsis in patients with dog bites. It is difficult to isolate due to culture growth requirements, which may explain the negative blood cultures in this case. Patients with alcoholism are predisposed to fulminant sepsis from $C$ canimorsus, which often presents with hepatic and renal failure. The myocardial ischemia may be secondary to the metabolic and thrombotic complications of sepsis.

On autopsy, there was purpura fulminans involving over $90 \%$ of the total body surface area as well as skin slippage and loose bullae of greater than $75 \%$ of the total body surface area. There was infarction of the kidneys, liver, spleen, and adrenal glands as well as focal contraction bands of necrosis of the myocardium. The lungs showed diffuse alveolar damage. There was hemorrhage, edema, and necrosis seen in sections taken from the puncture wounds. Following the patient's death, it was reported by the transferring institution that $C$ canimorsus was identified from 2 of 2 antemortem blood cultures, and pan-sensitive Acinetobacter lwoffii in 1 of 2 blood cultures, though no sensitivities were performed on the $C$ canimorsus isolate. In addition, ante- mortem cultures obtained at the academic medical center identified Capnocytophaga species in 1 of 2 peripheral blood culture specimens; sensitivities were not performed. Autopsy determined the cause of death in this patient to be septic complications of dog bite.

\section{COMMENTARY}

Dog bites are frequent, with over 12,000 occurring daily in the United States; of these, approximately 20\% require medical attention. ${ }^{1}$ Although most patients rapidly recover with conservative management, even initially benign-appearing injuries can lead to long-term morbidity or death. The hands are most often affected and are associated with more frequent need for both antibiotics and surgical intervention. ${ }^{2,3}$ The severity of injury does not correlate with subsequent infections. ${ }^{3}$

Management of dog bite injuries includes careful wound management. All patients with moderate to severe injury should be assessed within 48 hours by physical examination and radiography to assess the degree of injury and any associated nerve, tendon, joint, or bone damage. If there is concern for rabies based on history or vaccination status of the animal, prompt irrigation and debridement is crucial. Antimicrobial prophylaxis, typically with amoxicillin-clavulanate, should be given to high-risk patients, such as those with cirrhosis, asplenia, or other immunosuppressing conditions. ${ }^{4}$ Most infections are caused by Pasteurella and Bacteroides, whereas Capnocytophaga may cause severe disease, particularly in patients with immunosuppression or excessive alcohol intake. ${ }^{5}$ This patient was at increased risk of infection due to his late presentation following the initial bite and consequent delayed wound care, injury to the hand, and his history of alcoholism. ${ }^{4}$

Several members of the genus Capnocytophaga have been found in the oral cavities of both humans and canines. $C$ canimorsus, found only in canine or feline oral cavities, is the only member of the genus known to cause human disease. ${ }^{6}$ It is a fastidious gram-negative rod requiring an environment enriched with carbon dioxide, making it notoriously difficult to isolate. Cultures typically do not show growth for 5 to 7 days; thus, it is not surprising all cultures were initially negative in this case. ${ }^{4,7} \mathrm{C}$ canimorsus is a well-described cause of sepsis related to dog bites, with some cases bearing similarity to fulminant meningococcal disease. ${ }^{8}$ Severe illness typically occurs in immunosuppressed patients, particularly those with asplenia or cirrhosis. ${ }^{9,10}$ The pathophysiology of fulminant $C$ canimorsus infections is not well described. It has been suggested that certain strains may produce a toxin that inhibits macrophages and inactivates tumor necrosis factor in humans, although this is not yet widely accepted. ${ }^{11}$ Treatment of $C$ canimorsus involves early administration of effective antimicrobials, supportive care, and standard management of the bite injury. $C$ canimorsus is susceptible to several classes of antibiotics; $\beta$-lactams, such as penicillin derivatives and cephalosporins, and potentiated sulphonamides, such as trimethoprim/sulfamethoxazole, typically have the best in vitro activity. ${ }^{12}$ As illustrated in this case, even with prompt, effective antibiotic administration, $C$ canimorsus infection can progress to DIC, multisystem organ failure, and death. ${ }^{9}$

A lwoffii was also identified, but was almost certainly a contaminant. It is a gram-negative bacillus that is widely 
distributed throughout the environment. Commonly found on human skin and within the human oropharynx, it rarely causes human disease. Clinical manifestations of infection with A lwoffii are typically mild, and include superficial skin and soft tissue infection, urinary tract infection, and rarely bacteremia. Because of the severe presentation in this case and the compelling alternative explanation of $C$ canimorsus, A lwoffii was almost certainly a contaminant.

Rabies was an intriguing possibility in this case given the unvaccinated status of the dogs and the patient's prominent neurologic findings. Clinicians must consider the possibility of rabies in any patient with a bite injury from an unvaccinated dog. However, rabies remains extremely rare in the developed world as a result of the overwhelming success of animal vaccination and postexposure prophylaxis. Furthermore, rabies typically has an incubation period of several months. If rabies had caused this patient's presentation, rabies immunoglobulin would have been ineffective. Nevertheless, rabies prophylaxis with rabies immunoglobulin and vaccination is appropriate to prevent subsequent disease unless rabies infection can be definitively excluded. ${ }^{13}$

This patient presented with septic shock, DIC, and multisystem organ failure after a dog bite. The discussant quickly recognized the propensity of Capnocytophaga to cause this constellation of findings in alcoholic patients after dog bites. This patient did not have cirrhosis or asplenia, both of which are known risk factors for $C$ canimorsus infection; however, the fulminant presentation made $C$ canimorsus a necessary consideration. Ultimately, the dramatic nature of the patient's presentation combined with his history of heavy alcohol intake led the discussant to the correct diagnosis of septic shock secondary to $C$ canimorsus infection complicating a benign-appearing dog bite. Clinicians caring for patients who present with sepsis after a recent dog bite should consider C canimorsus, remembering that on occasion, a dog's bark may not be bigger than his bite.

\section{TEACHING POINTS}

1. The initial management of moderate or severe dogbite injuries includes careful wound assessment and radiography to exclude any associated bone, nerve, joint, or tendon injury.

2. Immunosuppressed patients with dog bites, including those with cirrhosis or asplenia, should receive amoxicillin/clavulanate prophylaxis.
3. C canimorsus is a fastidious gram-negative bacillus that may cause fulminant sepsis after dog bites. It is associated with DIC, purpura fulminans, and multisystem organ failure.

4. $\beta$-lactam antibiotics, such as penicillin derivatives or cephalosporins, or sulphonamides, are the treatment of choice for $C$ canimorsus.

Disclosure: Nothing to report.

\section{References}

1. Gilchrist J, Sacks JJ, White D, Kresnow MJ. Dog bites: still a problem? Injury Prev. 2008;14(5):296-301.

2. Pfortmueller CA, Efeoglou A, Furrer H, Exadaktylos AK. Dog bite injuries: primary and secondary emergency department presentations-a retrospective cohort study. ScientificWorldJournal. 2013; 2013:393176.

3. Akingba AG, Robinson EA, Jester AL, et al. Management of vascular trauma from dog bites. J Vascular Surg. 2013;58(5):1346-1352.

4. Morgan M, Palmer J. Dog bites. BMJ. 2007;334(7590):413-417.

5. Hermann CK, Hansen PB, Bangsborg JM, Pers C. Bacterial infections as complications of dog bites [in Danish]. Ugeskrift Laeger. 1998; 160(34):4860-4863.

6. Oehler RL, Velez AP, Mizrachi M, Lamarche J, Gompf S. Bite-related and septic syndromes caused by cats and dogs. Lancet Infect Dis. 2009;9(7):439-447.

7. Talan DA, Citron DM, Abrahamian FM, Moran GJ, Goldstein EJ. Bacteriologic analysis of infected dog and cat bites. Emergency Medicine Animal Bite Infection Study Group. N Engl J Med. 1999;340(2): $85-92$.

8. Janda JM, Graves MH, Lindquist D, Probert WS. Diagnosing Capnocytophaga canimorsus infections. Emerg Infect Dis. 2006;12(2):340342.

9. Pers C, Gahrn-Hansen B, Frederiksen W. Capnocytophaga canimorsus septicemia in Denmark, 1982-1995: review of 39 cases. Clinical Infect Dis. 1996;23(1):71-75.

10. Szabo G. Consequences of alcohol consumption on host defence. Alcohol Alcohol. 1999;34(6):830-841.

11. van Dam AP, van Weert A, Harmanus C, Hovius KE, Claas EC, Reubsaet FA. Molecular characterization of Capnocytophaga canimorsus and other canine Capnocytophaga spp. and assessment by PCR of their frequencies in dogs. J Clin Microbiol. 2009;47(10): 3218-3225.

12. Meyers B, Schoeman JP, Goddard A, Picard J. The bacteriology and antimicrobial susceptibility of infected and non-infected dog bite wounds: fifty cases. Vet Microbiol. 2008;127(3-4):360368 .

13. U.S. Department of Health and Human Services. Centers for Disease Control and Prevention. Human rabies-Alabama, Tennessee, and Texas, 1994. Morbidity and Mortality Weekly Report; 1995. Available at: http://www.cdc.gov/mmwr/preview/mmwrhtml/00036736. htm. Accessed March 1, 2014. 